Article

\title{
Study on Molecular Profiles of Staphylococcus aureus Strains: Spectrometric Approach
}

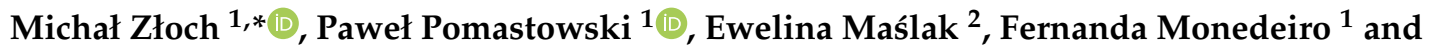 \\ Bogusław Buszewski 1,2 (D)
}

1 Centre for Modern Interdisciplinary Technologies, Nicolaus Copernicus University in Toruń, Wileńska 4 Str., 87-100 Toruń, Poland; p.pomastowski@umk.pl (P.P.); fernandamonedeiro@hotmail.com (F.M.); bbusz@chem.umk.pl (B.B.)

2 Chair of Environmental Chemistry and Bioanalytics, Faculty of Chemistry, Nicolaus Copernicus University in Toruń, Gagarina 7 Str., 87-100 Torun, Poland; ewelina.maslak@poczta.onet.pl

* Correspondence: michal.zloch@umk.pl; Tel.: +48-56-611-60-60

Received: 21 September 2020; Accepted: 18 October 2020; Published: 22 October 2020

check for updates

\begin{abstract}
Staphylococcus aureus remains a major health problem responsible for many epidemic outbreaks. Therefore, the development of efficient and rapid methods for studying molecular profiles of $S$. aureus strains for its further typing is in high demand. Among many techniques, matrix-assisted laser desorption ionization-time of flight mass spectrometry (MALDI TOF MS) represents a timely, cost-effective, and reliable strain typing approach, which is still rarely used due to insufficient knowledge about the impact of sample preparation and analysis conditions on the molecular profiles and strain classification efficiency of $S$. aureus. The aim of this study was to evaluate the effect of the culture conditions and matrix type on the differentiation of molecular profiles of various S. aureus strains via the MALDI TOF MS analysis and different computational methods. The analysis revealed that by changing the culture conditions, matrix type, as well as a statistical method, the differentiation of $S$. aureus strains can be significantly improved. Therefore, to accelerate the incorporation of the MALDI-based strain typing in routine laboratories, further studies on the standardization and searching of optimal conditions on a larger number of isolates and bacterial species are of great need.
\end{abstract}

Keywords: Staphylococcus aureus; spectrometric approach; strain typing; MALDI TOF MS; computational methods

\section{Introduction}

Staphylococcus aureus is a Gram-positive bacterium, the habitats of which are the nasal membranes and skin of warm-blooded animals. Due to secretion of extracellular factors and toxins as well as invasive properties, such as adherence, biofilm formation, and resistance to phagocytosis, it is the causative agent of many life-threatening invasive diseases [1]. Therefore, $S$. aureus remains a major health problem around the world since it is considered a leading cause of a wide range of hospitaland community-acquired infections [2-6]. Moreover, S. aureus demonstrates a high capability to adapt to a variety of conditions; therefore, its clones may spread very easily. The most "successful" ones (with enhanced virulence) can be responsible for an epidemic or even pandemic situation outbreaks [7]. Particularly important is rapid discrimination between methicillin-resistant (MRSA) and methicillin-susceptible $S$. aureus (MSSA) since the infection rate of MRSA in hospital settings has increased sharply, accounting for a huge number of deaths worldwide [8]. In order to detect and prevent such epidemic outbreaks, the clinical isolates have to be typed [2]. 
Among many techniques, the molecular typing of $S$. aureus isolates is believed to be an appropriate method for providing information about their phenotypic and genotypic variation at the strain level [9]. Currently, in addition to empirical biochemical tests, several molecular typing methods have been developed to understand epidemiology and pathogenicity of $S$. aureus strains in clinical diseases, e.g., pulsed-field gel electrophoresis (PFGE), randomly amplified polymorphic DNA (RAPD), multilocus sequence typing (MLST), or spa typing $[2,10]$. Although DNA-based techniques provide detailed subspecies information, they are labor-intensive, time-consuming, and cost-ineffective. Moreover, in the case of some of them (e.g., PFGE), achieving full reproducibility between laboratories is still a major challenge [11]. As the current challenge in diagnosing staphylococci and their drug-resistant varieties is the identification time, more recently, matrix-assisted laser desorption ionization-time of flight mass spectrometry (MALDI TOF MS), which has already been proven to be a rapid and reliable tool for identifying a wide array of microbial species [12], represents an alternative approach for timely, cost-effective, and reliable strain typing [11]. The principle of bacterial identification and classification at the genus and species level via the MALDI TOF MS technique is based on the generation of mass spectra representing specific molecular fingerprints of microorganisms composed of proteins that are surface-associated or extracted from whole cells, mostly ribosomal ones $[13,14]$. The success of the bacteria identification at the strain level proved more elusive compared to the species and genus levels due to the fact that members of a single species tended to yield remarkably similar MALDI TOF MS profiles. Still, in some cases, strains can also be differentiated based on subtle but reproducible differences $[15,16]$. Therefore, an increasing number of papers related to the utilization of MALDI TOF MS analysis for the subspecies discrimination of the microorganisms can be observed $[15,17,18]$. Several studies have hitherto demonstrated the great potential of this technique for discriminating bacteria at the subspecies level, e.g., distinguishing methicillin-resistant S. aureus strains (MRSA) from methicillin-sensitive $S$. aureus strains (MSSA), the distinction of biodiversity within S. epidermidis strains according to their origin, or subspecies typing of Salmonella enterica and Streptococcus pneumoniae strains [19-24]. The latest developments in this field include the detection of MRSA using the MALDI TOF mass spectrometry-based direct-on-target microdroplet growth assay, described as a novel method of phenotypic antimicrobial susceptibility testing [25].

There is wide variation in sample preparation regarding strain typing/profiling via the MALDI technique, e.g., intact cell method (ICM) [26] or protein extraction method (PEM) [27], liquid [28], or solid media [29]. Nevertheless, for all of them, the reproducibility and quality of spectra are of critical importance since the same bacterium may produce significantly different mass spectra under changing experimental conditions [30,31]. It is known that culture conditions, such as the composition of the growth medium, incubation time, $\mathrm{pH}$, or temperature, may lead to changes in the mass spectra profiles reflected in the variation of signal intensities, loss of signals, or the shift of signals probably caused by protein expression differences [2]. The impact of these factors should not be ignored since it can hinder the indication of mutations giving ambiguous information about a genotype of the strain. The reliable strain differentiation is also highly dependent on the procedures of the sample preparation and application onto the target plate as well as on the choice of the matrix, which affects the range of molecules being ionized, thus influencing the presence/absence of peaks $[32,33]$.

The utility of the MALDI approach at the strain level has not yet been completely explored [16] and there are not many works related to its application as a typing method for the classification of $S$. aureus strains [9]. In particular, extended research on experimental parameters' effects, such as the medium composition, type of matrix, or sample preparation procedure, is highly demanded, especially in the case of drug-resistant strains [29]. Moreover, inconsistent results have been obtained so far due to the fact that the spectra of highly related strains are similar and subtle differences among them are difficult to interpret by visual examination, which is still often used [17,34]. During phyloproteomic analysis, protein expression levels (signal intensities) are as important as the presence or absence of specific signals; thus, the interpretation of big data sets generated during MALDI analysis is complicated. Therefore, the usage of appropriate bioinformatics tools for the clustering of mass spectral data of 
proteins is recommended as a fast and efficient tool for the comparison of spectra $[9,16,35]$. For such purposes, many computational algorithms were developed, including cluster analysis (CA) [15], principle component analysis (PCA) [36], composite correlation index method (CCI) [37], or more recently machine learning (ML) methods, such as decision tree (DT) [11], support vector machine (SVM) [38], or the artificial neural network (ANN) [11,34]. Although some of them are included in the commercial software dedicated to microbial identification via the MALDI TOF MS technique, like in the case of the PCA, CCI, and CA available at the MALDI Biotyper Platform (Bruker Daltonics, US), their usefulness for the investigation of the impact of culture conditions on S. aureus profiling at the strain level is still poorly understood.

Therefore, the main purpose of this study was to evaluate the impact of culture medium composition along with the choice of the matrix on the molecular profiles of various $S$. aureus strains, including drug-resistant ones, analyzed by MALDI TOF MS analysis as a method of strain differentiation. Moreover, the strain profiling effectiveness obtained by various computational methods, such as hierarchical cluster analysis (HCA), PCA, and ANN, along with the visual inspection of phyloproteomic dendrograms via both the commercial software MALDI Biotyper 3.0 (Bruker Daltonics, US) and the self-developed algorithms in the R environment [39] was compared and evaluated.

\section{Results}

\subsection{Impact of Culture Conditions and Matrices on the Identification Level}

The analysis of the score values revealed that the impact of the culture medium composition is highly dependent on the matrix used as well as the type of MS data: raw spectra or MSP (Table 1). Exemplary MS spectra obtained for different matrices are presented in Figure 1.

The variance of score values was in the range 1.76-2.36 (RAW data) or 1.65-2.35 (MSP). Based on the raw MS data, a significant influence of the culture medium type was revealed only when the HCCA was used except for strains cultured on the MAN medium, which demonstrated the lowest identification levels regardless of the matrix used $(1.76 \pm 0.18-1.90 \pm 0.15)$. In the HCCA variants, bacteria cultured on two media, BLA and CHRA, were characterized by the highest score values ( $2.25 \pm 0.13$ and $2.27 \pm 0.14$, respectively) followed by all the universal media and the BHI. The VRE medium obtained significantly lower score values; however, the identification was at the species level $(2.04 \pm 0.07)$. In turn, the culturing of $S$. aureus strains on the MAN medium allowed only for good genus identification $(<2.00)$. The application of the other two matrices did not reveal differences between culture media with the exception of the MAN, which, as in the case of the HCCA, was characterized by a considerably lower identification level (decrease by an average of 0.49 score value). Moreover, in the case of the universal media, BHI, and VRE, the use of DHB or SDHB significantly improved the identification quality compared to the HCCA one: by 0.16 on the MHA to $0.20-0.25$ of score value on the VRE medium.

In the case of five media, the MHA, TSA, BHI, BLA, and VRE, a significant impact of the matrix on the number of signals in the MS spectra was observed (Table 2).

Except the BLA medium, a significantly higher number of signals were detected when the DHB or SDHB was used: from two to six more peaks. Generally, the DHB matrix was characterized by the richest MS spectra (90-100 signals) followed by the SDHB (86-100) and the HCCA (84-99). Considering the culture media, the CHRA and MAN demonstrated the lowest number of peaks: less by $6-15,1-10$, or 3-14 signals on the HCCA, DHB, and SDHB, respectively. Regarding the MSP data, the results for MAN medium were worse independently of the matrix used. However, a similar decline in the identification level quality was observed. Nevertheless, the same effect of the DHB and SDHB on the identification quality improvement was noted. 
Table 1. Impact of culture medium and type of matrix on the identification level of investigated S. aureus strains expressed as an average score value (the logarithm of the result of matching the investigated spectrum with patterns in the reference database) calculated from the results of 4 repetitions for each variant of the experiment (mean \pm standard deviation). Statistical significance of differences was tested using one-way ANOVA (ANalysis Of VAriance) with post-hoc Tukey HSD (Honestly Significant Difference) post-hoc test. The influence of the medium is marked in large font while the small font is used to indicate differences between matrices.

\begin{tabular}{cccccc}
\hline \multicolumn{5}{c}{ RAW Data } \\
\hline Medium & HCCA & DHB & SDHB & General & \\
\hline MHA & $2.09 \pm 0.12 \mathrm{Bb}$ & $2.27 \pm 0.13 \mathrm{Aa}$ & $2.27 \pm 0.09 \mathrm{Aa}$ & $2.21 \pm 0.14$ & \\
TSA & $2.11 \pm 0.09 \mathrm{Bb}$ & $2.29 \pm 0.12 \mathrm{Aa}$ & $2.26 \pm 0.09 \mathrm{Aa}$ & $2.22 \pm 0.13$ & universal \\
COL & $2.13 \pm 0.11 \mathrm{Bb}$ & $2.31 \pm 0.14 \mathrm{Aa}$ & $2.34 \pm 0.11 \mathrm{Aa}$ & $2.26 \pm 0.15$ & \\
\hline BHI & $2.07 \pm 0.09 \mathrm{Bb}$ & $2.29 \pm 0.11 \mathrm{Aa}$ & $2.27 \pm 0.11 \mathrm{Aa}$ & $2.21 \pm 0.14$ & enriched \\
BLA & $2.25 \pm 0.13 \mathrm{Aa}$ & $2.36 \pm 0.12 \mathrm{Aa}$ & $2.27 \pm 0.20 \mathrm{Aa}$ & $2.31 \pm 0.17$ & \\
\hline CHRA & $2.27 \pm 0.14 \mathrm{Aa}$ & $2.30 \pm 0.11 \mathrm{Aa}$ & $2.18 \pm 0.18 \mathrm{Aa}$ & $2.24 \pm 0.16$ & differentiating \\
\hline VRE & $2.04 \pm 0.07 \mathrm{Cb}$ & $2.24 \pm 0.15 \mathrm{Aa}$ & $2.29 \pm 0.15 \mathrm{Aa}$ & $2.19 \pm 0.17$ & selective \\
MAN & $1.90 \pm 0.15 \mathrm{Da}$ & $1.80 \pm 0.17 \mathrm{Ba}$ & $1.76 \pm 0.18 \mathrm{Ba}$ & $1.82 \pm 0.18$ & \\
\hline & & MSP data & & \\
\hline MHA & $1.94 \pm 0.12 \mathrm{Ab}$ & $2.17 \pm 0.18 \mathrm{Aa}$ & $2.18 \pm 0.11 \mathrm{Aa}$ & $2.07 \pm 0.18$ & \\
TSA & $2.12 \pm 0.11 \mathrm{Ab}$ & $2.30 \pm 0.11 \mathrm{Aa}$ & $2.26 \pm 0.09 \mathrm{Aa}$ & $2.20 \pm 0.13$ & universal \\
COL & $2.07 \pm 0.10 \mathrm{Ab}$ & $2.29 \pm 0.12 \mathrm{Aa}$ & $2.35 \pm 0.24 \mathrm{Aa}$ & $2.21 \pm 0.20$ & \\
\hline BHI & $2.00 \pm 0.12 \mathrm{Ab}$ & $2.22 \pm 0.12 \mathrm{Aa}$ & $2.23 \pm 0.08 \mathrm{Aa}$ & $2.13 \pm 0.15$ & enriched \\
BLA & $2.12 \pm 0.16 \mathrm{Aa}$ & $2.25 \pm 0.33 \mathrm{Aa}$ & $2.22 \pm 0.21 \mathrm{Aa}$ & $2.17 \pm 0.25$ & \\
\hline CHRA & $2.10 \pm 0.18 \mathrm{Aa}$ & $2.21 \pm 0.12 \mathrm{Aa}$ & $2.14 \pm 0.21 \mathrm{Aa}$ & $2.13 \pm 0.18$ & differentiating \\
\hline VRE & $2.01 \pm 0.11 \mathrm{Ab}$ & $2.19 \pm 0.15 \mathrm{Aa}$ & $2.23 \pm 0.12 \mathrm{Aa}$ & $2.12 \pm 0.16$ & \multirow{2}{*}{ selective } \\
MAN & $1.77 \pm 0.21 \mathrm{Ba}$ & $1.69 \pm 0.21 \mathrm{Ba}$ & $1.65 \pm 0.24 \mathrm{Ba}$ & $1.68 \pm 0.22$ & \\
\hline
\end{tabular}

TSA-Tryptic Soy Agar, MHA-Mueller Hinton Agar, COL-Columbia Agar Base, BHI-Brain Heart Infusion Agar, BLA-Columbia Blood Agar, CHRA-CHROMagarTM Orientation, MAN-Mannitol Salt Agar, VRE-Vancomycin Resistant Enterococci Agar Base, HCCA- $\alpha$-Cyano-4-hydroxycinnamic acid, DHB-2,5-dihydroxybenzoic acid, SDHB-mixture of DHB and 2-hydroxy-5-methoxybenzoic acid (9:1 w/w), MSP-main spectrum (peak lists generated from raw spectra by algorithms included in MALDI Biotyper), RAW data-Score values obtained for the whole spectra, MSP data-Score values obtained for main spectra.

Table 2. The average number of signals detected in RAW spectra obtained for investigated S. aureus strains cultured on the different media depending on the matrix used. * statistically higher based on the one-way ANOVA (ANalysis of VAriance) with post-hoc Tukey HSD (Honestly Significant Difference) post-hoc test regarding matrix effect.

\begin{tabular}{cccc}
\hline \multirow{2}{*}{ Medium } & \multicolumn{3}{c}{ Matrix } \\
\cline { 2 - 4 } & HCCA & DHB & SDHB \\
\hline MHA & 93 & $98^{*}$ & $98^{*}$ \\
TSA & 98 & $100^{*}$ & $100^{*}$ \\
COL & 97 & 99 & 99 \\
BHI & 93 & $98^{*}$ & $99 *$ \\
BLA & $99 *$ & 92 & 93 \\
CHRA & 87 & 91 & 90 \\
VRE & 94 & $100^{*}$ & $98^{*}$ \\
MAN & 84 & 90 & 86 \\
\hline
\end{tabular}

TSA-Tryptic Soy Agar, MHA-Mueller Hinton Agar, COL-Columbia Agar Base, BHI-Brain Heart Infusion Agar, BLA-Columbia Blood Agar, CHRA-CHROMagarTM Orientation, MAN-Mannitol Salt Agar, VRE-Vancomycin Resistant Enterococci Agar Base, HCCA- $\alpha$-Cyano-4-hydroxycinnamic acid, DHB-2,5-dihydroxybenzoic acid, SDHB-mixture of DHB and 2-hydroxy-5-methoxybenzoic acid (9:1 w/w). 

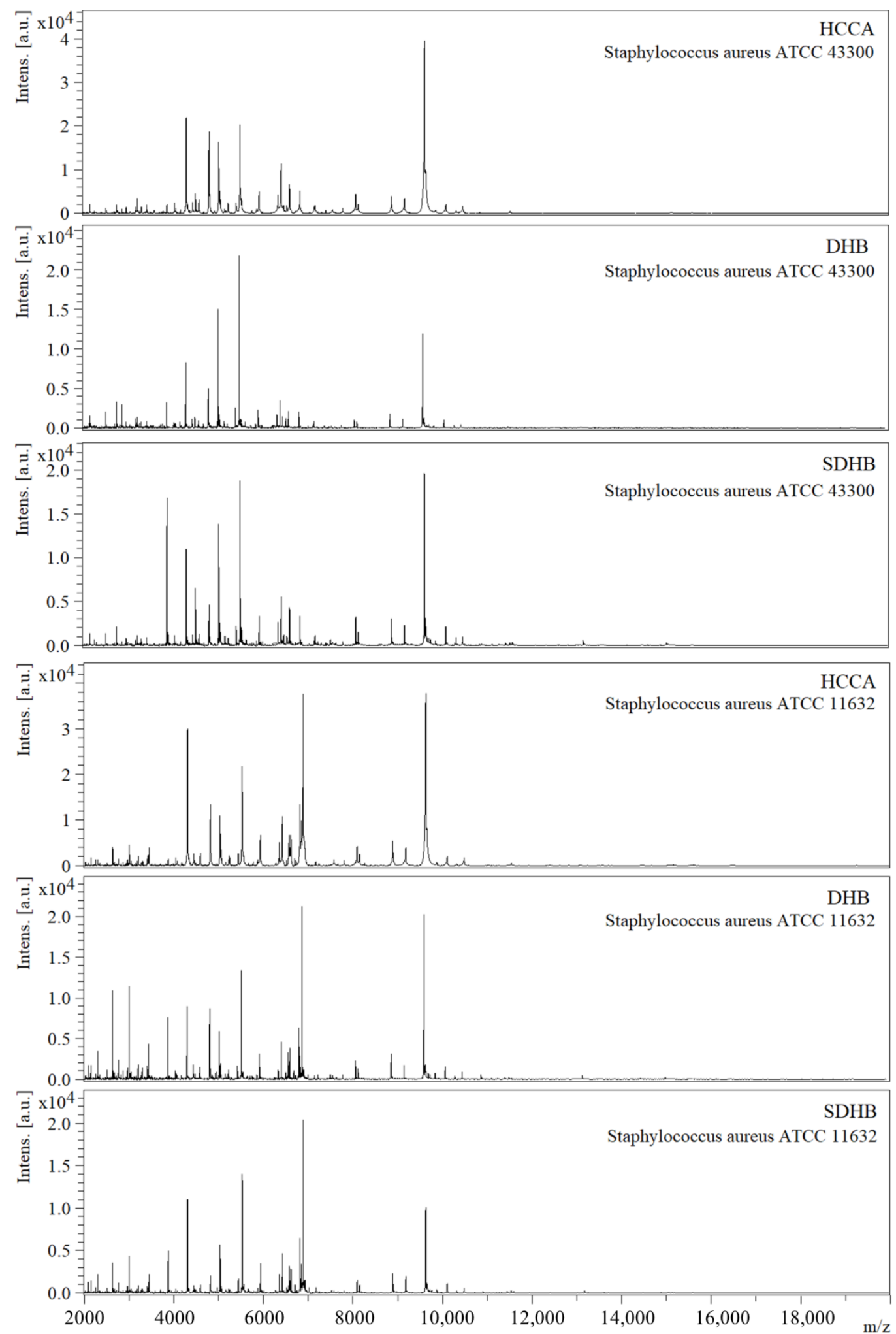

Figure 1. Exemplary MS profiles of the investigated $S$. aureus strains generated during MALDI TOF MS analysis using different matrices: HCCA ( $\alpha$-Cyano-4-hydroxycinnamic acid), DHB (2,5-dihydroxybenzoic acid), and SDHB (mixture of DHB and 2-hydroxy-5-methoxybenzoic acid (9:1 w/w). BLA-Columbia Blood Agar. 


\subsection{Analysis of Discriminatory Power Depending on the Culture Medium and Matrix Using the MALDI Biotyper Platform}

Based on the obtained MSPs (main spectra), created by the transformation of raw spectra into peak lists by extracting information on peak mass, peak frequency, and peak intensity distribution using the Biotyper Preprocessing Standard Method, phyloproteomic trees were generated. The visual inspection of the MSP dendrograms revealed a significant impact of both the culture medium composition and the matrix on the S. aureus classification capacities (Table 3).

Table 3. Comparison of classification capacities obtained for individual media and 3 different matrices using main spectra (MSPs) dendrogram analysis (via MALDI Biotyper 3.0) as well as hierarchical cluster analysis (HCA) and principal components analysis (PCA) via R software expressed as a percentage share of correctly classified strains among 7 investigated S. aureus strains. An increase in the color intensity in the column means a higher value for the parameter.

\begin{tabular}{ccccccccccccc}
\hline \multicolumn{1}{c}{ Classification Capacities Values [\%] Calculated for all Investigated Strains } \\
\hline \multicolumn{1}{c}{$\begin{array}{c}\text { MSPs Dendrograms Analysis } \\
\text { Matrix }\end{array}$} & \multicolumn{3}{c}{$\begin{array}{c}\text { HCA Analysis } \\
\text { Matrix }\end{array}$} & \multicolumn{3}{c}{ PCA Analysis } \\
Matrix
\end{tabular}

MSP-main spectrum (peak lists generated from raw spectra by algorithms included in MALDI Biotyper), TSA-Tryptic Soy Agar, MHA-Mueller Hinton Agar, COL-Columbia Agar Base, BHI-Brain Heart Infusion Agar, BLA-Columbia Blood Agar, CHRA-CHROMagarTM Orientation, MAN-Mannitol Salt Agar, VRE-Vancomycin Resistant Enterococci Agar Base, HCCA- $\alpha$-Cyano-4-hydroxycinnamic acid, DHB-2,5-dihydroxybenzoic acid, SDHB-mixture of DHB and 2-hydroxy-5-methoxybenzoic acid (9:1 w/w).

Considering the matrix effect, the best results were obtained using the HCCA (52\% general correct classification), which in the case of five media gave the highest percentage of correct strain groupings: $43-71 \%$. Only for the COL and CHRA media, a better classification was observed in the DHB or SDHB variants, respectively. In the case of one medium, mannitol salt agar (MAN), no matrix effect was noted: a $57 \%$ classification capacity for all investigated matrices. Regarding the culture media, the best discrimination of $S$. aureus strains was noted for enriched and selective media; in both cases, the percentage of correct classification ranged from $52-57 \%$. Taking into account both investigated parameters, the highest classification capacity was observed for variants of BHI, BLA, and VRE + HCCA along with COL + DHB- 71\%. In terms of drug-resistant strains' grouping, the classification capacities were higher as compared to the analysis that also included sensitive ones by $10-13 \%$ regarding matrices among which the HCCA was similarly the best one. Comparing a different 
type of culture media, enriched and selective ones were also characterized by the highest percentage of correct classifications: $66-77 \%$ and 55-66\%, respectively. As with the analysis performed including all S. aureus strains, the classification of drug-resistant strains cultured on the MAN and BLA media were not affected by the type of the matrix used: $66 \%$ in each case.

Additionally, the evaluation of the strain discrimination capacity of the MALDI Biotyper 3.0 platform was performed based on the correlation values derived from the CCI (composite correlation index method) analysis of raw spectra data. The analysis of the differences between the degree of similarity within the MS spectra of individual strains to that noted between various strains revealed a great impact of the culture medium composition, which differed depending on the matrix used (Table 4).

Table 4. CCI analysis results showing the differences between the degree of similarity within the strain (intrastrain) to that noted between the strains (interstrain) of the MS spectra of the tested $S$. aureus strains depending on the medium and matrix used presented as a result of their subtraction $(\Delta)$. An increase in the color intensity in the column means a higher value for the parameter.

\begin{tabular}{ccccc}
\hline & \multicolumn{4}{c}{$\begin{array}{c}\text { Difference Between the Intrastrain and } \\
\text { Interstrain Degree of Similarity }(\boldsymbol{\Delta})\end{array}$} \\
Medium & HCCA Matrix & DHB Matrix & SDHB Matrix & Mean \\
\hline MHA & 0.12 & 0.16 & 0.07 & $\mathbf{0 . 1 2}$ \\
TSA & 0.06 & 0.10 & 0.05 & $\mathbf{0 . 0 7}$ \\
COL & 0.12 & 0.15 & 0.14 & $\mathbf{0 . 1 4}$ \\
BHI & 0.12 & 0.19 & 0.09 & $\mathbf{0 . 1 3}$ \\
BLA & 0.26 & 0.17 & 0.20 & 0.21 \\
CHRA & 0.15 & 0.15 & 0.25 & $\mathbf{0 . 1 8}$ \\
VRE & 0.18 & 0.13 & 0.18 & $\mathbf{0 . 1 6}$ \\
MAN & 0.10 & 0.06 & 0.11 & $\mathbf{0 . 0 9}$ \\
\hline
\end{tabular}

TSA-Tryptic Soy Agar, MHA-Mueller Hinton Agar, COL-Columbia Agar Base, BHI-Brain Heart Infusion Agar, BLA-Columbia Blood Agar, CHRA-CHROMagarTM Orientation, MAN-Mannitol Salt Agar, VRE-Vancomycin Resistant Enterococci Agar Base, HCCA- $\alpha$-Cyano-4-hydroxycinnamic acid, DHB-2,5-dihydroxybenzoic acid, SDHB-mixture of DHB and 2-hydroxy-5-methoxybenzoic acid $(9: 1 w / w)$.

The best discrimination between $S$. aureus strains was obtained using the BLA + HCCA and $\mathrm{SDHB}, \mathrm{CHRA}+\mathrm{SDHB}$, or BHI + DHB where differences between intra- and interstrain similarities $(\Delta)$ reached $0.26,0.20,0.25$, and 0.19 , respectively. In general, the best classification was noted for the BLA, CHRA, and VRE while the use of bacterial cells cultured on the TSA and MAN media gave the worst results. Comparing matrices, the best matrix for all universal media and BHI agar was DHB, while in the case of selective ones, better classification was achieved using HCCA and SDHB.

\subsection{Analysis of Discriminatory Power Depending on the Culture Medium and Matrix Using R Environment}

As with the results of the analysis performed via the MALDI Biotyper platform, the investigation of the classification capacity using the $\mathrm{R}$ software revealed a great impact of both the matrix and the culture medium (Table 3). Depending on the statistical method used for the data assessment, the percentage of correct $S$. aureus strains' classification was significantly higher (the hierarchical cluster analysis using Spearman's method, HCA) or lower (the principal component analysis, PCA) compared to the visual inspection of MSP dendrograms. This phenomenon regarded both analyses of all the strains and the drug-resistant ones only.

Considering the HCA, the analysis revealed a considerable improvement of the overall classification capacity when the HCCA matrix was used: $84 \%$ compared to 52\%. On five media, TSA, BLA, CHRA, VRE, and MAN, a 100\% good strain classification was achieved (exemplary heatmaps presented in Figure 2A). The worst discriminatory power level (43\%) was achieved using MHA medium. Regarding the other two matrices, a significant decline in the overall classification capacity in comparison to the HCCA matrix was noted; however, their levels were still higher compared to that obtained using the Biotyper platform. The differences in the discriminatory power between both matrices were only slightly different and depended on the culture medium composition. For DHB, 
discrimination power was better when TSA or MAN cultures were applied (Figure 2B) while the SDHB was superior for the BLA and MAN variants (Figure 2C).

A

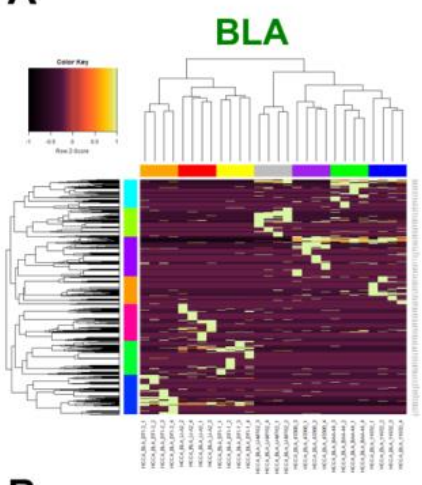

B

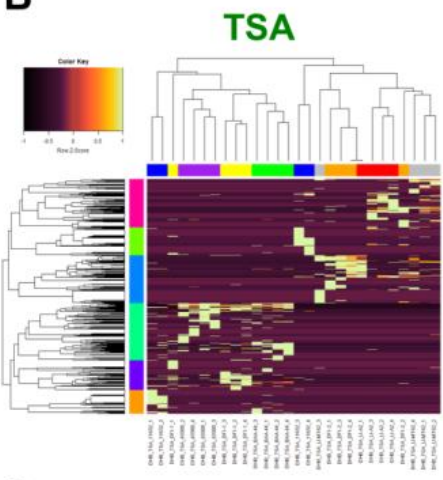

C

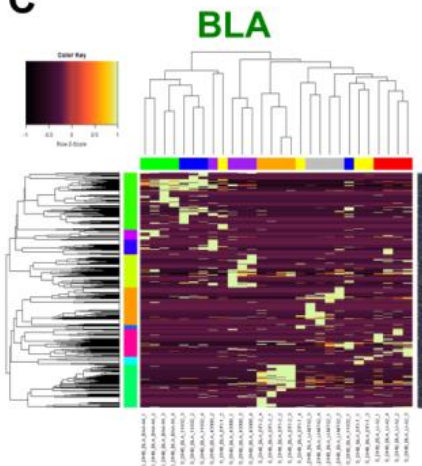

Legend (horizontal colours)

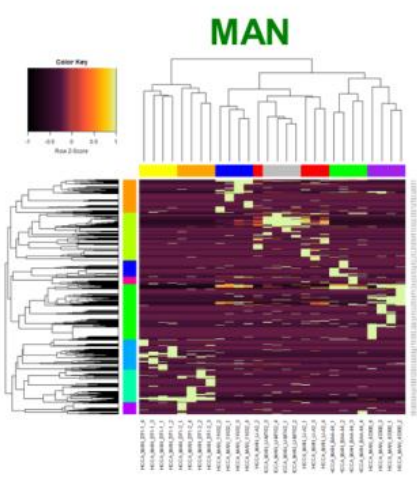

MAN

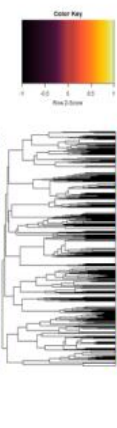

MAN
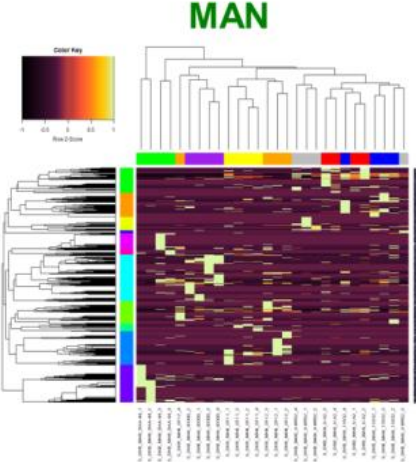
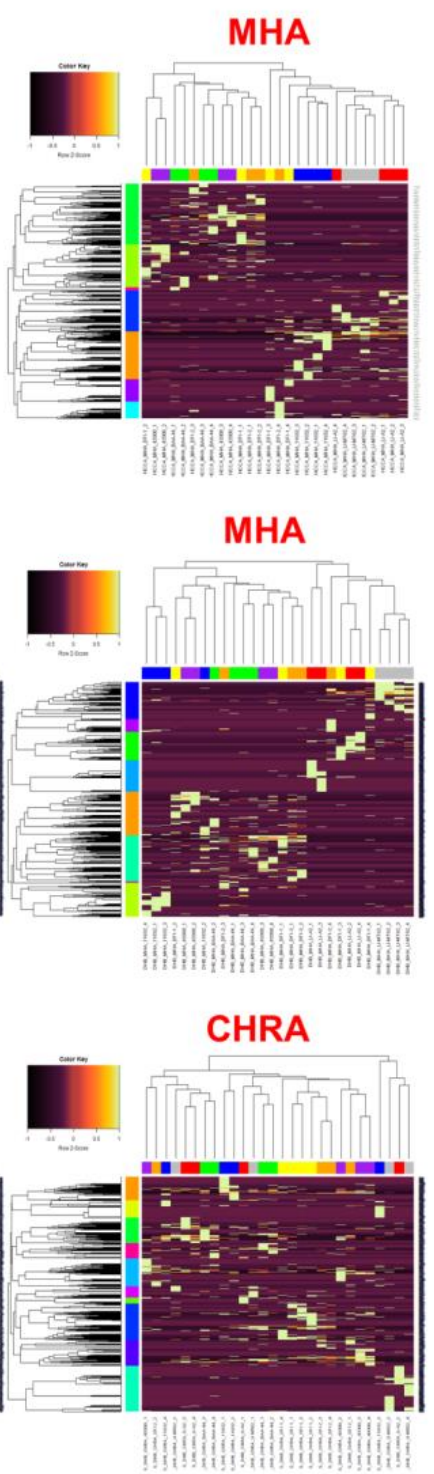

$\square 43300$

BAA-44

11632

$\square$ DFI-1

DFI-2

$\mathrm{U}-\mathrm{A} 2$

U-MT62

Figure 2. The best (green font) and worst (red font) results of classification of S. aureus strains using the hierarchical cluster analysis (HCA) depending on the culture medium and matrix ((A) HCCA matrix, (B) DHB matrix, (C) SDHB matrix) performed using Spearman clustering method based on the average values. TSA-Tryptic Soy Agar, MHA-Mueller Hinton Agar, BLA-Columbia Blood Agar, CHRA-CHROMagar ${ }^{\mathrm{TM}}$ Orientation, MAN-Mannitol Salt Agar, HCCA- $\alpha$-Cyano-4-hydroxycinnamic acid, DHB-2,5-dihydroxybenzoic acid, SDHB-mixture of DHB and 2-hydroxy-5-methoxybenzoic acid $(9: 1 w / w)$.

The lowest strain classification capacities were noted for MHA (DHB) or CHRA (SDHB) bacteria cultures. For the MHA medium, no influence of the matrix was noted. Taking into account the results obtained for all the matrices, the best classification was achieved using the MAN, TSA, or BLA medium at $86 \%, 81 \%$, and $76 \%$ of the general capacity, respectively (Table 3). The worst results of $S$. aureus grouping were observed for the CHRA, COL (both $52 \%$ ), as well as for bacteria cultured on the MHA medium 
(43\%). Considering the classification of drug-resistant strains, the mean percentage of the correct grouping on the HCCA was similar to that obtained for all tested strains at $83 \%$, while in the case of the other two matrices, better results were achieved by 8-11\% (Table 3). Regarding the culture composition, the best general classification effectiveness was obtained on the MAN medium-100\% regardless of the matrix used-followed by the BLA (78\%), TSA, and VRE at 77\% for both.

The application of the PCA method demonstrated the lowest discriminatory power among all the approaches applied (Figure 3) and in relation to the most effective method, HCA, the overall classification capacities on the HCCA, DHB, and SDHB were 2.2, 2.4, and 3 times lower. Considering all matrices, three culture media were characterized by the best strain discrimination: BLA, MAN, and VRE.

A
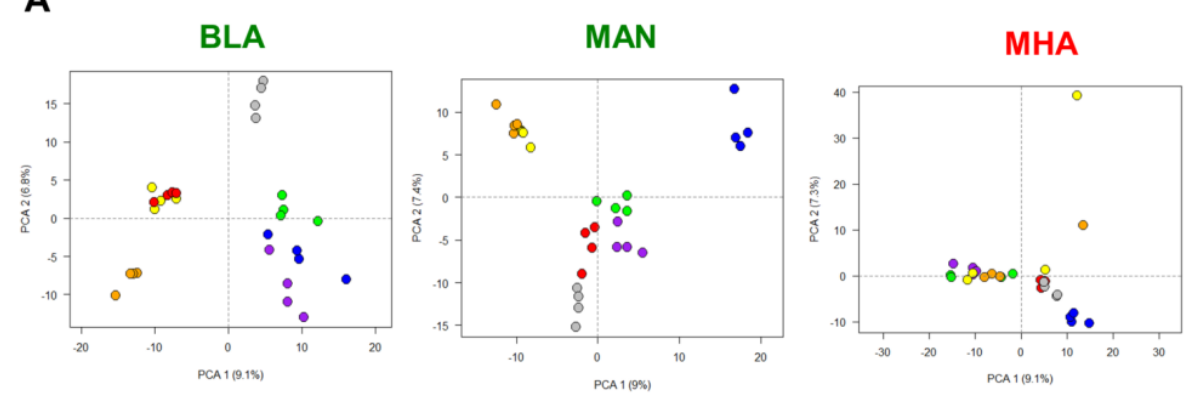

B
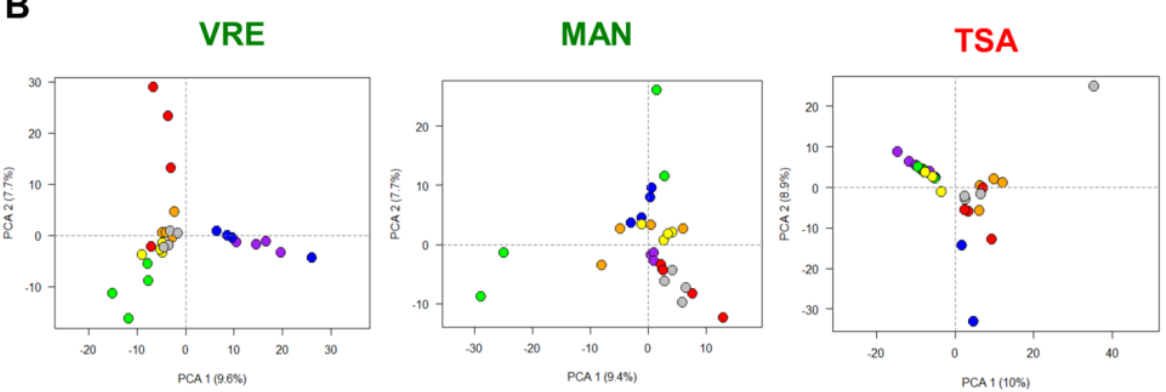

C
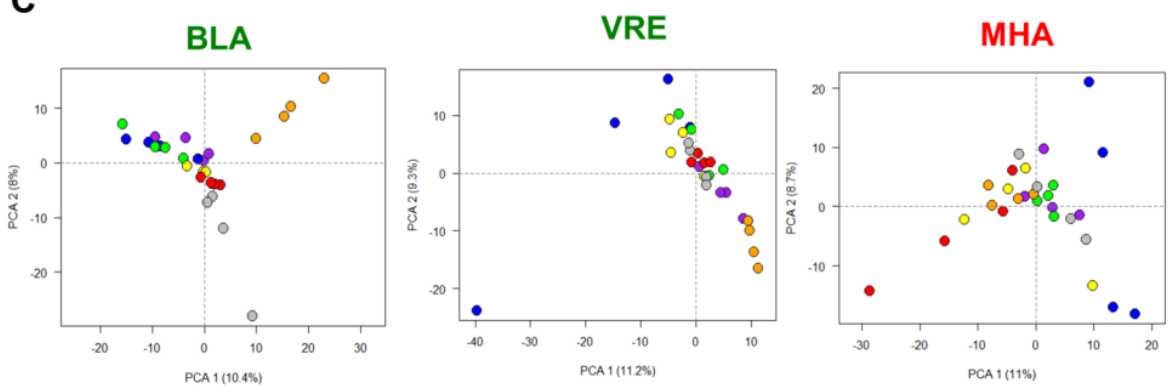

Legend (horizontal colours)

$\square 43300 \square$ BAA-44

$\square 11632$

DFI-1

DFI-2

$\mathrm{UU}-\mathrm{A} 2$

U-MT62

Figure 3. The best (green font) and worst (red font) results of classification of $S$. aureus strains using principal component analysis (PCA) depending on the culture medium and matrix ((A) HCCA, (B) DHB, (C) SDHB). Data with only $>10 \%$ of total abundance was used. TSA-Tryptic Soy Agar, MHA-Mueller Hinton Agar, BLA-Columbia Blood Agar, MAN-Mannitol Salt Agar, VRE-Vancomycin Resistant Enterococci Agar Base, HCCA- $\alpha$-Cyano-4-hydroxycinnamic acid, DHB-2,5-dihydroxybenzoic acid, SDHB-mixture of DHB and 2-hydroxy-5-methoxybenzoic acid (9:1 w/w).

In five cases, TSA or $\mathrm{BHI}+\mathrm{DHB}$, and $\mathrm{MHA}$, TSA, or BHI + SDHB, no good classification was noted (Table 3). Comparing the culture media, the best strains grouping was achieved using MAN 
(48\% general capacity), VRE (43\%), or BLA (38\%), while the application of MHA, TSA, or BHI resulted in the poorest discrimination at $14 \%$ and $10 \%$ of the general classification capacity, respectively. Similar, in the case of drug-resistant strains, their classification using the PCA technique was considerably lower as compared to that obtained via HCA analysis. Significantly, the best grouping was obtained using the MAN medium at $66 \%$ of the general classification effectiveness compared to the $44 \%$ achieved for the second-best medium, VRE or COL. In the case of one medium, BHI, no correct classification was observed.

The obtained ANN models allowed for S. aureus strains' classification with an accuracy in the test data (TDA) at the level of at least $90 \%$ in half of the cases while only four variants demonstrated TDA below $80 \%$ (Table 5).

Table 5. Obtained artificial neural network (ANN) models used to predict the classification of investigated $S$. aureus strains via $\mathrm{R}$ software depending on the culture medium and matrix used. Hidden layers $=3,10$-fold cross validation testing $95 \%$ of data per sampling, data partition $=0.5$. TTA-accuracy in the train test; TDA-accuracy in the test data; Model ions-ions with discriminating features used to create model.

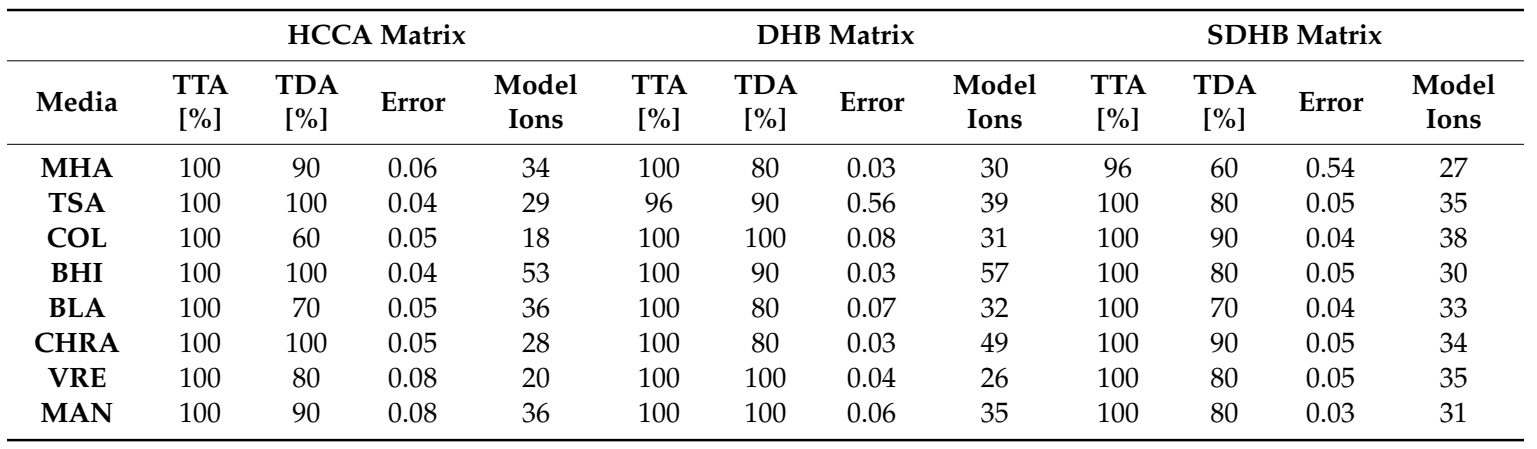

TSA-Tryptic Soy Agar, MHA-Mueller Hinton Agar, COL-Columbia Agar Base, BHI-Brain Heart Infusion Agar, BLA-Columbia Blood Agar, CHRA-CHROMagarTM Orientation, MAN-Mannitol Salt Agar, VRE-Vancomycin Resistant Enterococci Agar Base, HCCA- $\alpha$-Cyano-4-hydroxycinnamic acid, DHB-2,5-dihydroxybenzoic acid, SDHB-mixture of DHB and 2-hydroxy-5-methoxybenzoic acid (9:1 w/w).

Like for the other analytical methods used, the matrix and culture medium composition significantly influenced the prediction accuracy of the created ANN models. Although the application of the DHB gave the highest TDA values for four media as in the case of HCCA, in general, the use of this matrix provided the best accuracy, which did not fall below $80 \%$. Like in the other methods, the ANN models created on the basis of the results obtained using the SDHB were characterized by the worst prediction accuracy among all the investigated matrices. Considering the culture composition, four media, TSA, BHI, CHRA, and MAN, had the highest TDA values followed by the VRE and COL. Interestingly, the worst prediction was achieved for the culture media both relatively poor in nutrients (MHA) and the enriched ones (BLA). In the six combinations of matrix and culture medium, ANN models were characterized by 100\% TDA-HCCA + TSA, BHI, or CHRA and DHB + COL, VRE, or MAN. Regarding the number of ions required for obtaining the optimized model, they significantly differed between variants and ranged from 18 to 57. Comparing matrices, the highest number of ions were noted for the DHB: on average, 37. In the case of the other two matrices, HCCA and SDHB, a similar average number of ions was disclosed, 32 and 33, respectively, but variation within the SDHB models was smaller (27-38 compared to 18-53 when the HCCA was used). Considering the culture media, the lowest number of model ions was noted for VRE (20-35) followed by COL (18-38) and MHA (27-34) while the use of results derived from BHI variants demanded easily the highest number of model ions among all the investigated media at 30-57. Taking into account both the prediction accuracy, number of model ions, as well as the error of the prediction, the best ANN models were obtained using the VRE + DHB followed by CHRA + HCCA and TSA + HCCA. 
The results of the mass spectra repeatability analysis expressed as a percentage of relevant correlations between corresponding MS replicates calculated using the Spearman correlation method revealed a great impact of the culture media composition, while the matrix effect was ambiguous (Table 6).

Table 6. Percentage of relevant correlations between corresponding replicates of mass spectra measured for individual $S$. aureus strains depending on the matrix and culture medium calculated using the Spearman correlation method. NC: significant correlation between non-corresponding assays). An increase in the color intensity in the column means a higher value for the parameter.

\begin{tabular}{cccccccccc}
\hline & BAA-44 & $\mathbf{4 3 3 0 0}$ & $\mathbf{1 1 6 3 2}$ & U-A2 & U-MT62 & DFI-1 & DFI-2 & NC* & General \\
\hline \multicolumn{7}{c}{ Relevant } & Correlations [\%] between Replicates-HCCA Matrix & & \\
\hline MHA & 16.67 & 33.33 & 0.00 & 16.67 & 50.00 & 0.00 & 16.67 & 0.00 & $\mathbf{1 9}$ \\
TSA & 83.33 & 66.67 & 100.00 & 66.67 & 50.00 & 50.00 & 50.00 & 0.40 & $\mathbf{6 7}$ \\
COL & 16.67 & 0.00 & 16.67 & 50.00 & 100.00 & 16.67 & 33.33 & 1.59 & $\mathbf{3 3}$ \\
BHI & 16.67 & 16.67 & 83.33 & 0.00 & 83.33 & 16.67 & 50.00 & 2.38 & $\mathbf{3 8}$ \\
BLA & 83.33 & 83.33 & 66.67 & 16.67 & 100.00 & 0.00 & 100.00 & 0.00 & $\mathbf{6 4}$ \\
CHRA & 66.67 & 100.00 & 100.00 & 0.00 & 50.00 & 50.00 & 33.33 & 0.79 & $\mathbf{5 7}$ \\
VRE & 33.33 & 16.67 & 16.67 & 50.00 & 33.33 & 16.67 & 16.67 & 0.00 & $\mathbf{2 6}$ \\
MAN & 16.67 & 100.00 & 33.33 & 33.33 & 100.00 & 0.00 & 16.67 & 1.19 & $\mathbf{4 3}$ \\
\hline \multicolumn{7}{c}{ Relevant Correlations [\%] between Replicates-DHB Matrix } & & \\
\hline MHA & 0.00 & 33.33 & 33.33 & 16.67 & 100.00 & 0.00 & 16.67 & 0.40 & $\mathbf{2 9}$ \\
TSA & 83.33 & 66.67 & 33.33 & 50.00 & 16.67 & 50.00 & 50.00 & 1.98 & $\mathbf{5 0}$ \\
COL & 16.67 & 16.67 & 16.67 & 50.00 & 16.67 & 16.67 & 33.33 & 1.59 & $\mathbf{2 4}$ \\
BHI & 16.67 & 16.67 & 66.67 & 0.00 & 50.00 & 0.00 & 50.00 & 1.19 & $\mathbf{2 9}$ \\
BLA & 16.67 & 33.33 & 16.67 & 66.67 & 33.33 & 16.67 & 100.00 & 2.38 & $\mathbf{4 0}$ \\
CHRA & 50.00 & 16.67 & 66.67 & 33.33 & 16.67 & 66.67 & 16.67 & 1.59 & $\mathbf{3 8}$ \\
VRE & 16.67 & 33.33 & 83.33 & 33.33 & 33.33 & 50.00 & 33.33 & 0.79 & $\mathbf{4 0}$ \\
MAN & 16.67 & 83.33 & 100.00 & 33.33 & 50.00 & 50.00 & 0.00 & 0.99 & $\mathbf{4 8}$ \\
\hline \multicolumn{8}{c}{ Relevant Correlations [\%] between Replicates-SDHB Matrix } & & \\
\hline MHA & 0.00 & 16.67 & 16.67 & 33.33 & 33.33 & 0.00 & 50.00 & 8.33 & $\mathbf{2 1}$ \\
TSA & 33.33 & 66.67 & 0.00 & 83.33 & 0.00 & 83.33 & 100.00 & 10.32 & $\mathbf{5 2}$ \\
COL & 50.00 & 66.67 & 66.67 & 16.67 & 16.67 & 16.67 & 100.00 & 1.59 & $\mathbf{4 8}$ \\
BHI & 50.00 & 50.00 & 100.00 & 50.00 & 33.33 & 50.00 & 16.67 & 14.68 & $\mathbf{5 0}$ \\
BLA & 50.00 & 50.00 & 33.33 & 50.00 & 50.00 & 16.67 & 100.00 & 1.19 & $\mathbf{5 0}$ \\
CHRA & 83.33 & 33.33 & 16.67 & 33.33 & 16.67 & 50.00 & 16.67 & 3.57 & $\mathbf{3 6}$ \\
VRE & 83.33 & 33.33 & 16.67 & 33.33 & 16.67 & 50.00 & 16.67 & 3.57 & $\mathbf{3 6}$ \\
MAN & 0.00 & 33.33 & 66.67 & 83.33 & 0.00 & 66.67 & 16.67 & 1.59 & $\mathbf{3 8}$ \\
\hline
\end{tabular}

TSA-Tryptic Soy Agar, MHA-Mueller Hinton Agar, COL-Columbia Agar Base, BHI-Brain Heart Infusion Agar, BLA-Columbia Blood Agar, CHRA-CHROMagarTM Orientation, MAN-Mannitol Salt Agar, VRE-Vancomycin Resistant Enterococci Agar Base, HCCA- $\alpha$-Cyano-4-hydroxycinnamic acid, DHB-2,5-dihydroxybenzoic acid, SDHB-mixture of DHB and 2-hydroxy-5-methoxybenzoic acid (9:1 w/w), NC*-significant correlation between non-corresponding assays.

Taking into account the effect of the culture medium, significantly, the highest repeatability of MS spectra was noted for TSA (50-67\%) and BLA (40-64\%) followed by CHRA (36-57\%) and MAN $(38-48 \%)$. Considerably lower repeatability was observed for the poorest MHA medium at $19-29 \%$. Regarding the type of matrix used, their impact depended on the culture medium; in the case of TSA, BLA, and CHRA, the highest repeatability was achieved using HCCA $(67 \%$, 64\%, and 57\%, respectively), while DHB was better in the case of MAN, VRE, and MHA. Nevertheless, the use of none of the matrices improved the general repeatability.

\section{Discussion}

The identification of bacteria to the species and genus level via the MALDI TOF MS technique is generally considered independent of culture conditions since most of the detected proteins are ribosomal ones. It is believed they represent ca. $50 \%$ of the peaks of all bacteria that can be unambiguously 
associated with ribosomal proteins while the presence of the rest, they are more or less metabolic status dependent [40]. Therefore, in the literature, the question was raised whether bacterial cultures growing on rich culture media can be used to identify bacteria rather than poorer ones [41]. The results of our studies revealed only a slight impact of the media composition on the identification level. However, all the media except the mannitol salt agar achieved score values $\geq 2.000$, recognized as a high confidence level. It proved the statement that the effect of the media composition on the MALDI TOF mass spectral quality is minimal with no impact on the species identification [42]. The MAN medium was characterized by considerably lower score values allowed only for the secure genus identification, which may result from the interference of the medium composition with the ionization of bacterial biomolecules, e.g., sodium chloride at very high concentration $(75 \mathrm{~g} / \mathrm{L})$. Nevertheless, the low identification level noted for the MAN medium could also be explained by the difficulty in obtaining a sufficient amount of cell biomass $\left(5 \times 10^{5}\right.$ cells on a target spot $)$ since the bacteria were strongly attached on the surface of this agar and demonstrated low growth at the same time. A similar phenomenon was observed for Arcobacter, Helicobacter, and Campylobacter strains grown on mCCD agar in the work of Alispahic et al. [43]. However, this issue relates more to the effect of the type of culture medium on the growth rate of the bacteria than on the differences in the composition of the protein profile itself. Surprisingly, in most cases, the application of the HCCA decreased identification scores, especially for universal media, although this matrix is most widely used and recommended for routine analysis. In the work of Dieckmann et al. [20], during testing of three different matrices, DHB, HCCA, and sinapinic acid (SA), HCCA appeared to have been a better choice than DHB since it enabled the detection of more peaks and provided more homogeneous crystallization. Such features are important in so-called common use, because the DHB matrix during crystallization creates needle-shaped crystals that hinder its use in an automated routine analysis. Although HCCA is known to be more efficient in ionization and thus gives a higher signal intensity, DHB produces fewer background signals from matrix clusters, which could be responsible for better score values in the manual measurement mode [44].

In contrast with the identification level, the culture media composition demonstrated a great impact on the S. aureus classification correctness. It indicates that the composition of non-ribosomal proteins, which are metabolic status dependent, play a significant role in the discrimination between close-related bacterial strains. Moreover, differences between the effect of culture media significantly changed depending on the matrix and even the method used for the classification capacity evaluation. It is known that the spectra of the same bacterial species obtained in different conditions demonstrated large variations, which influence the reproducibility and quality of the MALDI TOF results. It emphasizes the need for standardization of the sample preparation like the choice of matrix or culture medium, especially in view of subspecies differentiation [45]. It may result from the fact that in the case of subspecies differentiation, a much larger number of reproducible peaks is needed in comparison to those usually required for the identification of microbial isolates [20]. The most significant differences between the results of the classification capacities of the investigated $S$. aureus strains were observed between the most minimal universal MHA medium and both enriched Columbia blood agar and highly selective mannitol salt agar. The first one was characterized by the worst classification of the strains regardless of the matrix and the calculation method used while the other two media were the best ones. As was noted in the work of Vargha et al. [33], beside the matrix, differences in the growth media and growth stage of bacteria may also generate variations in the MALDI TOF MS spectra. Yet, keeping growth conditions constant could resolve this problem, but there is a risk that some valuable physiological information may be overlooked. Carbonelle et al. [46], who investigated the impact of both the MHA and Columbia blood agar on the identification of staphylococci isolated in clinical microbiology laboratories, observed that some peaks varied with the growth conditions. On the other hand, Bernardo et al. [47] observed no dramatic influence of the growth media on the MALDI profiles in the case of $S$. aureus strains cultured on MHA or Columbia blood agar. However, both studies related to the identification level. As our studies showed, the subtle differences between the culture media composition did not influence the identification significantly. Nevertheless, they considerably changed 
the differentiation capacity of close-related strains. Generally, the use of enriched or selective enhanced the correct classification of investigated strains as compared to the universal ones. Interestingly, the application of the MAN medium, which enabled only the secure genus identification, allowed good strain differentiation regardless of the calculation method used. Moreover, in the case of resistant strains, it turned out to be the best choice, giving $100 \%$ correct classifications on each matrix when considering the HCA results (Table 3). The MAN medium is both a selective and differential medium for culturing staphylococci on which $S$. aureus can be easily identified from samples with little or no contamination [48]. Therefore, it could be used for fast and accurate strain differentiation in those clinical specimens where $S$. aureus is expected to be the dominant causative agent. Moreover, one of the best classification capacities was obtained using bacteria cultured on the Columbia blood agar (BLA). Considering that strain differentiation using the Columbia agar base alone (COL) demonstrated a significantly lower effectiveness compared to that one with sheep blood addition, it can be deduced that such conditions induced some new metabolic pathways in bacteria, leading to the appearance of more discriminant signals. As our study showed, the induction of additional metabolic pathways may in result in the improvement of the differentiation of closed-related species. Blood agar is commonly used in routine clinical laboratories as a standard medium for the cultivation of clinical isolates, especially fastidious ones. In view of this, the implementation of strain typing using cultures cultivated on blood agar in epidemiological studies should be easily feasible. However, some authors pointed out the problem with the use of blood-containing media related to the contamination of the MS profiles with blood-related proteins like 15,048 and 16,075 m/z along with their doubly charged variants as was the case in the work of Dieckmann et al. [20] about Salmonella species and subspecies classification where Mueller-Hinton agar was chosen as the standard medium for routine analysis. Nevertheless, the responsibility for such a phenomenon could be the use of the intact cells mode instead of their protein extracts like it was in our studies, where we did not observe interferences originating from blood-related proteins. Goldstein et al. [29] observed a significant influence of the sample preparation mode on $S$. aureus strain profiling, where PEM appeared to be the better one. The same authors observed significant differences comparing solid and liquid media. The above-mentioned studies along with our findings indicate that by changing the metabolic needs that different culturing conditions present and by choosing the sample preparation technique (e.g., ICM or PEM), we can improve or deteriorate the classification of strains. Moreover, the identification of the bacteria to the genus or species level and strain typing should be considered separately, using other criteria for the evaluation of the created protocols.

The choice of the matrix had no less effect on the ability to distinguish between the tested S. aureus strains than the choice of the medium. Interestingly, HCCA, which was characterized by the lowest identification quality, turned out to be the best for the strain discrimination. However, its advantage over the other two matrices was smaller or larger depending on the applied evaluation method. Regarding the unsupervised methods, the best overall strain differentiation ability for the HCCA variant was achieved when the HCA method was used, 84\%, a value almost one third higher than the discriminating ability obtained when the DHB or SDHB matrices were used. A similar phenomenon was also noted for resistant strains. The HCCA demonstrated enhanced ionization properties, leading to higher peak intensities and thus a large number of the signals in MS profiles, an important factor during identification at the strain level, which usually requires a large number of reproducible peaks. Since HCCA is more preferred for peptides and smaller proteins than DHB or SDHB [44], it is also suggested that signals belonging to higher $\mathrm{m} / \mathrm{z}$ values played a less important role in the discrimination of the tested S. aureus strains than smaller ones. Moreover, Pomastowski et al. [49] observed that the different nature of matrix crystallization resulted in a different extraction mechanism of bacterial components, e.g., DHB could be more favorable for the analysis of lipoproteins, whereas HCCA in the case of DNA-binding proteins. Considering this, in-depth studies on the effect of the matrix on strain typing seems to be useful in searching for characteristic signals that could be used as biomarkers for distinguishing close-related species in the near future. 
Although the reproducibility of the MS profiles is considered to be one of the crucial factors affecting strain differentiation [29], in our studies, their values did not vary significantly between matrices; therefore, it is likely that such an aspect was not the main factor responsible for differences in the classification capabilities. Nevertheless, the results of the strain discrimination strongly depended on the method used for their evaluation. Over 10 years ago, researchers pointed out that the application of the MALDI TOF MS demonstrated a highly accurate method for bacterial classification if it was provided with a suitable model construction [50] since the strain discrimination involved the generation of substantial and complex datasets [16], causing the visual inspection of spectra to become inadequate to obtain accurate strain differentiation [51]. Until now, many different algorithms (commercial or in-house software solutions), including the peak intensity or the binary peak list (absence or presence) in the calculation of similarities/dissimilarities between spectra, have been applied and several discrepancies in the literature regarding the taxonomic limits can be found [52]. Although even single minor software features can have profound effects on the ability of the MALDI TOF MS to discriminate bacterial strains, it should be noted that contradictory results may also be caused by the use of diverse sample preparation techniques. Both phenomena were observed in our studies and played an important role in the discrimination of the investigated S. aureus strains. The HCA based on the correlation analysis appeared to be the better tool to discriminate $S$. aureus strains than PCA, which is related to a summarized variance of the variables. It may result from a relatively low reproducibility of replicates, especially from a quantitative point of view. In the case of HCA, we chose the Spearman rank correlation method, recommended for non-parametric data, when variables from different assays appear to be related between each other by a monotonic nonlinear relationship [53]. Since a higher correlation between replicates was achieved by using this approach, it can be concluded that the variables from corresponding replicates covary. In PCA, a new system of coordinates is created, corresponding to the set of variables with higher variance in the system. In the HCA process, a measurement of similarity between paired samples is conducted [54,55]. It means that variables did not display prominent behavior in the overall system, which may be responsible for the similarity within the group. This aspect indicates that the segregation according to strains' groups occurred more on the basis of the incidence of ions rather than their quantitative indexes (ions intensities). Our results indicate that the HCA method demonstrates great potential for the discrimination of closely related species once the employment of appropriate conditions for the cultivation of microorganisms is taken into account. Similarly, the HCA was successfully applied in the classification of human pathogenic bacteria, where they categorized the spectra into six groups precisely corresponding to the six bacterial species even when the numbers of $m / z$ values were reduced to six [50]. In turn, Lasch et al. [3] evaluated the discriminatory power of the MALDI TOF MS technique for typing Enterococcus faecium and S. aureus isolates and concluded that the HCA provided insufficient subspecies classification. However, the authors did not exclude that the observed limited ability for differentiation and identification of clonal complexes of E. faecium and S. aureus might result from inappropriate culture conditions. Indeed, it seems that our research confirms the explanation put forward by the authors. Moreover, the use of different parameters for correlation calculation, like Euclidean distances between bar code spectra and limiting the signals to the 50 peaks list, could also matter.

The prediction accuracy of $S$. aureus strains via designed ANN models was significantly affected by the culture medium composition and, to a lesser extent, by the matrix choice as well. Nevertheless, this method demonstrates the best subspecies discrimination among all used methods. Machine learning methods, including the ANN, have become of particular interest to researchers regarding subspecies differentiation in view of developing a robust approach based on the MALDI TOF spectrum. In the recent literature, we can find studies confirming the high utility of the ML predictive models combined with the MALDI technique in the discrimination of such species as S. haemolyticus or S. aureus [11,38]. The implementation of machine learning-based methods seems to solve the problem of analyzing complex proteomic data, giving high-throughput and appropriate precision. However, even this approach appeared to be affected by the culture media composition and matrix, which should not be 
ignored while searching for optimal MALDI solutions for subspecies discrimination. To our knowledge, the presented work is the first to address the impact of the culture medium composition and the matrix on the final prediction accuracy of the ANN models based on the MALDI mass spectra in view of bacteria differentiation at the strain level. Since the presented studies used analysis in the linear mode (mostly applied in the clinical laboratories), it could also be promising to investigate the selected conditions using the reflectron mode (TOF/TOF MS), which could both help explain mechanisms underlying the revealed phenomena and give the opportunity to select specific peaks for improving S. aureus differentiation. However, this represents a different approach with other criteria to be considered, like, for example, the lower intensity of the characteristic peaks (biomarkers), and will be the subject of further research.

\section{Materials and Methods}

\subsection{Investigated Strains}

During the experiment, 7 different Staphylococcus aureus strains were used: Three strains derived from the American Type Culture Collection (ATCC): (1) S. aureus subsp. aureus Rosenbach ATCC 43,300 (clinical isolate, Kansas; methicillin and oxacillin resistant; MRSA SCCmec: Type II); (2) S. aureus subsp. aureus Rosenbach ATCC 11,632 (penicillin resistant); as well as (3) S. aureus subsp. aureus Rosenbach ATCC BAA-44 (Iberian clone of MRSA SCCmec: Type I isolated from a hospital in Lisbon, Portugal; Multidrug-resistant strain); all strains were purchased as Kwik-Stik (Pol-Aura, Poland). The remaining four strains were clinical isolates obtained from the urine of healthy volunteers: S. aureus U-A2 and S. aureus U-MT62; as well as from diabetic foot infections: S. aureus DFI-1 and S. aureus DFI-2.

\subsection{Culture Conditions}

During study implementation, 8 different solid culture media were used: (A) universal-Tryptic Soy Agar (TSA; Sigma Aldrich, Steinheim, Germany), Mueller Hinton Agar (MHA; Sigma Aldrich, Steinheim, Germany), Columbia Agar Base (COL; Oxoid, Basingstoke, UK); (B) enriched-Brain Heart Infusion Agar (BHI; Sigma Aldrich, Steinheim, Germany); Columbia Blood Agar (BLA; Oxoid, Basingstoke, UK); (C) differentiating chromogenic medium-CHROMagar ${ }^{\mathrm{TM}}$ Orientation (CHRA; GRASO Biotech, Starogard Gdanski, Poland) as well as (D) selective-Mannitol Salt Agar (MAN; Oxoid, Basingstoke, UK), and Vancomycin Resistant Enterococci Agar Base (VRE; Oxoid, Basingstoke, UK). All media were in the form of ready-to-use powders except for BLA, which was prepared by adding defibrinated sheep blood (GRASO Biotech, Starogard Gdanski, Poland) to the sterilized and dissolved Colombia blood agar base to the final concentration $5 \%(v / v)$. Before analysis, all investigated S. aureus strains were cultured on plates containing each solid medium at $37^{\circ} \mathrm{C}$ in aerobic conditions by $24 \mathrm{~h}$.

\subsection{MALDI TOF MS Analysis}

For sample preparation, the protein extraction method according to Bruker's guideline was used. From one-day cultures of $S$. aureus, 1 inoculation loop $(10 \mu \mathrm{L})$ of biomass was transferred into $300 \mu \mathrm{L}$ of sterile deionized water, $900 \mu \mathrm{L} \mathrm{96 \%} \mathrm{ethanol} \mathrm{was} \mathrm{added,} \mathrm{and} \mathrm{after} \mathrm{mixing} \mathrm{centrifuged} \mathrm{(13,000} \mathrm{RPM,}$ $5 \mathrm{~min}$.). The supernatant was removed, and the remaining cell pellet was dried in a vacuum centrifuge at room temperature. Then, dried cell pellet was subjected to formic acid (FA)/acetonitrile (ACN) extraction using $10 \mu \mathrm{L} 70 \%$ FA and $10 \mu \mathrm{L}$ ACN. After mixing, the sample extract was centrifuged (13,000 RPM, 5 min.) and $1 \mu \mathrm{L}$ of supernatant was transferred onto a MALDI MTP 384 ground steel target sample spot (Bruker Daltonik GmbH, Bremen, Germany). After air-drying, the sample spot was overlaid with $1 \mu \mathrm{L}$ of respective matrix solution: (1) $\alpha$-Cyano-4-hydroxycinnamic acid (HCCA): $10 \mathrm{mg} / \mathrm{mL}$ in standard solvent solution (50\% ACN, $47.5 \%$ water and $2.5 \%$ trifluoro acetic acid); (2) 2,5-dihydroxybenzoic acid (DHB): $50 \mathrm{mg} / \mathrm{mL}$ or (3) SuperDHB (9:1 (w/w) mixture of DHB and 2-hydroxy-5-methoxybenzoic acid, respectively): $50 \mathrm{mg} / \mathrm{mL}$ in standard solvent solution. MALDI Target 
plates with the samples were analyzed using ultrafleXtreme MALDI-TOF/TOF mass spectrometer (Bruker Daltonik GmbH, Bremen, Germany) equipped with the smartbeam-II laser-positive mode according to the procedure described in detail in a previous work [56]. Spectra were collected manually using manufacturer software, flexControl, and the following parameters: $\mathrm{m} / \mathrm{z}$ range: $2000-20,000$, acceleration voltage at $25 \mathrm{kV}$, global attenuator offset at $20 \%$ and attenuator offset at $34 \%$ and its range at $34 \%$, laser power at $40 \%, 500$ shots in-one-single spectra to frequency 2500 . Obtained spectra were subjected to smoothing using the Savistsky-Golay method (width $2 \mathrm{~m} / \mathrm{z}$, cycles 10) and baseline corrections using the TopHat algorithm (signal to noise threshold 2; peak detection algorithm-centroid) recommended by the software supplier (Bruker Daltonik $\mathrm{GmbH}$, Bremen, Germany) as well as calibration with BTS (Bruker Bacterial Test Standard, Bruker Daltonik GmbH, Bremen, Germany) in quadratic mode using the manufacturer software, flexAnalysis. Each sample representing an individual strain cultured on a specific medium and ionized using a particular matrix was measured in quadruplicate.

\subsection{Spectra Analysis Using MALDI Biotyper 3.0 Platform}

All spectra were subjected to the species identification via the MALDI Biotyper 3.0 platform based on the both raw spectra (RAW) and Main Spectra (MSP) peaks lists created by the transformation of raw spectra by extracting information on peak mass, peak frequency, and peak intensity distribution using the Biotyper Preprocessing Standard Method recommended and provided by the manufacturer (Bruker Daltonik GmbH, Bremen, Germany). Based on the MSP spectra, phyloproteomic dendrograms were generated to determine the classification capacity of individual S. aureus strains depending on the culture medium and matrix by visual inspection. Classification capacity was understood as the ability to separate MSP spectra into separate clusters according to the individual strain type, expressed as a percentage of correctly grouped strains. A correctly classified strain was considered to have at least 3 of 4 spectra grouped together as a separate cluster. The general classification capacity for each of the tested matrices was calculated for each strain as a percentage of correct classifications obtained on all tested media. Next, the total classification capacity for a given strain was calculated and expressed as the average value of classification abilities obtained on each of the tested matrices.

\subsection{Self-Developed Statistical and Chemometric Approaches for Spectra Analysis}

Primary data consisted of spectra's 100 most abundant ions and their respective intensities (relative intensity of the peak in relation to the spectrum of the calibration standard, BTS). In R environment (using RStudio console v. 1.1.463), data referring to each experiment were converted in data frames and joined into a single database presenting ion $(\mathrm{m} / \mathrm{z})$ and intensities. In a first approach, the level of correspondence between ion profiles obtained from replicates of a given assay were tested using Spearman correlation analysis, processed using IBM SPSS Statistics v.24. A correlation coefficient $<0.4$ and with a $p$ value $\leq 0.05$ was considered as the relevance criteria to assume correspondence between the tested profiles. Then, unsupervised inspection of data was conducted in R. Hierarchical cluster analysis was performed using Spearman's method, and heatmaps were generated using Z-score scaling of the input data. Principal component analysis (PCA) was also conducted to verify the distribution of profiles according to the strains in each medium, input data comprehended scaled intensities, and data with only $>10 \%$ of total abundance was included. For generation of the latter graphical representations, the "gplots" package was used. Artificial neural networks (ANNs) were performed in order to develop a multiclass classificatory model, able to predict bacterial strains according to the presence or absence of a set of ions. The discriminating features (ions), were selected as those that provided a lower $p$ value when the Mann-Whitney U test was applied in an approach "single class vs. all others". The number of selected variables was empirically verified as the minimum to assure greater model performance. Input for ANN consisted of a primary database with intensities converted into binary entries. The $\mathrm{R}$ packages "neuralnet" and "nnet" were employed in this step and 3 hidden layers were used. A 10-fold cross validation testing $95 \%$ of the data per sampling was performed to assess model accuracy. 


\section{Conclusions}

Over the past decade, the MALDI TOF MS technique has become a reliable tool for fast and robust microbial identification that revolutionized the workflow of clinical laboratories. Currently, due to advancements in dedicated software, computational algorithms, as well as the greater availability of the devices (lower prices), the application of the MALDI approach for microbial identification at the subspecies level is under insightful debate. Unfortunately, there is a lack of in-depth studies conducted on the role of the culture conditions or the matrix choice on the molecular profiles of S. aureus strains obtained by MALDI TOF MS analysis and their usefulness for strain classification. The results of our study showed that by changing culture conditions or by choosing a different matrix type, we can both improve or worsen the differentiation of S. aureus strains. Moreover, the choice of the statistical method also significantly affects the strain clustering among which the HCA and ANN models demonstrated the most promising application. Our work emphasizes the great need for further studies on a larger number of isolates and other bacterial species. Such studies may accelerate the incorporation of the MALDI-based subspecies identification approach in routine laboratories in the future.

Author Contributions: Conceptualization, M.Z. and P.P.; methodology, M.Z.; software, F.M.; validation, M.Z., P.P. and F.M.; formal analysis, M.Z.; investigation, E.M.; resources, M.Z.; data curation, F.M.; writing—original draft preparation, M.Z.; writing - review and editing, P.P.; visualization, F.M.; supervision, B.B.; project administration, M.Z.; funding acquisition, M.Z. All authors have read and agreed to the published version of the manuscript.

Funding: This research was funded by National Science Centre, Poland, grant number 2018/28/C/ST4/00434 (Sonatina 2). The APC was funded by National Science Centre, Poland, grant number 2018/28/C/ST4/00434 (Sonatina 2).

Acknowledgments: M.Z., P.P., E.M. and B.B. are members of Torun Center of Excellence “Towards Personalized Medicine" operating under Excellence Initiative-Research University.

Conflicts of Interest: The authors declare no conflict of interest. The funders had no role in the design of the study; in the collection, analyses, or interpretation of data; in the writing of the manuscript, or in the decision to publish the results.

\section{References}

1. Chessa, D.; Ganau, G.; Mazzarello, V. An overview of Staphylococcus epidermidis and Staphylococcus aureus with a focus on developing. J. Infect. Dev. Ctries. 2015, 9, 547-550. [CrossRef] [PubMed]

2. Josten, M.; Reif, M.; Szekat, C.; Al-Sabti, N.; Roemer, T.; Sparbier, K.; Kostrzewa, M.; Rohde, H.; Sahl, H.G.; Bierbaum, G. Analysis of the matrix-assisted laser desorption ionization-time of flight mass spectrum of Staphylococcus aureus identifies mutations that allow differentiation of the main clonal lineages. J. Clin. Microbiol. 2013, 51, 1809-1817. [CrossRef] [PubMed]

3. Lasch, P.; Fleige, C.; Stämmler, M.; Layer, F.; Nübel, U.; Witte, W.; Werner, G. Insufficient discriminatory power of MALDI-TOF mass spectrometry for typing of Enterococcus faecium and Staphylococcus aureus isolates. J. Microbiol. Methods 2014, 100, 58-69. [CrossRef] [PubMed]

4. Østergaard, C.; Hansen, S.G.K.; Møller, J.K. Rapid first-line discrimination of methicillin resistant Staphylococcus aureus strains using MALDI-TOF MS. Int. J. Med. Microbiol. 2015, 305, 838-847. [CrossRef] [PubMed]

5. Goudarzi, M.; Seyedjavadi, S.S.; Nasiri, M.J.; Goudarzi, H.; Sajadi Nia, R.; Dabiri, H. Molecular characteristics of methicillin-resistant Staphylococcus aureus (MRSA) strains isolated from patients with bacteremia based on MLST, SCCmec, spa, and agr locus types analysis. Microb. Pathog. 2017, 104, 328-335. [CrossRef]

6. Asadollahi, P.; Farahani, N.N.; Mirzaii, M.; Khoramrooz, S.S.; van Belkum, A.; Asadollahi, K.; Dadashi, M.; Darban-Sarokhalil, D. Distribution of the most prevalent spa types among clinical isolates of methicillin-resistant and -susceptible Staphylococcus aureus around the world: A review. Front. Microbiol. 2018, 9, 163. [CrossRef]

7. Parhizgari, N.; Khoramrooz, S.S.; Malek Hosseini, S.A.A.; Marashifard, M.; Yazdanpanah, M.; Emaneini, M.; Gharibpour, F.; Mirzaii, M.; Darban-Sarokhalil, D.; Moein, M.; et al. High frequency of multidrug-resistant Staphylococcus aureus with SCCmec type III and Spa types t037 and t631 isolated from burn patients in southwest of Iran. APMIS 2016, 124, 221-228. [CrossRef] 
8. Kim, J.-M.; Kim, I.; Chung, S.H.; Chung, Y.; Han, M.; Kim, J.-S. Rapid Discrimination of Methicillin-Resistant Staphylococcus aureus by MALDI-TOF MS. Pathogens 2019, 8, 214. [CrossRef]

9. Böhme, K.; Morandi, S.; Cremonesi, P.; Fernández No, I.C.; Barros-Velázquez, J.; Castiglioni, B.; Brasca, M.; Cañas, B.; Calo-Mata, P. Characterization of Staphylococcus aureus strains isolated from Italian dairy products by MALDI-TOF mass fingerprinting. Electrophoresis 2012, 33, 2355-2364. [CrossRef]

10. Sabat, A.; Malachowa, N.; Miedzobrodzki, J.; Hryniewicz, W. Comparison of PCR-based methods for typing Staphylococcus aureus isolates. J. Clin. Microbiol. 2006, 44, 3804-3807. [CrossRef] [PubMed]

11. Wang, H.-Y.; Lee, T.-Y.; Tseng, Y.-J.; Liu, T.-P.; Huang, K.-Y.; Chang, Y.-T.; Chen, C.-H.; Lu, J.-J. A new scheme for strain typing of methicillin-resistant Staphylococcus aureus on the basis of matrix-assisted laser desorption ionization time-of-flight mass spectrometry by using machine learning approach. PLoS ONE 2018, 13, e0194289. [CrossRef] [PubMed]

12. Ge, M.C.; Kuo, A.J.; Liu, K.L.; Wen, Y.H.; Chia, J.H.; Chang, P.Y.; Lee, M.H.; Wu, T.L.; Chang, S.C.; Lu, J.J. Routine identification of microorganisms by matrix-assisted laser desorption ionization time-of-flight mass spectrometry: Success rate, economic analysis, and clinical outcome. J. Microbiol. Immunol. Infect. 2017, 50, 662-668. [CrossRef] [PubMed]

13. Sauer, S.; Kliem, M. Mass spectrometry tools for the classification and identification of bacteria. Nat. Rev. Microbiol. 2010, 8, 74-82. [CrossRef]

14. Doan, N.T.L.; Van Hoorde, K.; Cnockaert, M.; De Brandt, E.; Aerts, M.; Le Thanh, B.; Vandamme, P. Validation of MALDI-TOF MS for rapid classification and identification of lactic acid bacteria, with a focus on isolates from traditional fermented foods in Northern Vietnam. Lett. Appl. Microbiol. 2012, 55, 265-273. [CrossRef]

15. Wolters, M.; Rohde, H.; Maier, T.; Belmar-Campos, C.; Franke, G.; Scherpe, S.; Aepfelbacher, M.; Christner, M. MALDI-TOF MS fingerprinting allows for discrimination of major methicillin-resistant Staphylococcus aureus lineages. Int. J. Med. Microbiol. 2011, 301, 64-68. [CrossRef] [PubMed]

16. Sandrin, T.R.; Goldstein, J.E.; Schumaker, S. MALDI TOF MS profiling of bacteria at the strain level: A review. Mass Spectrom. Rev. 2013, 32, 188-217. [CrossRef] [PubMed]

17. Camoez, M.; Sierra, J.M.; Dominguez, M.A.; Ferrer-Navarro, M.; Vila, J.; Roca, I. Automated categorization of methicillin-resistant Staphylococcus aureus clinical isolates into different clonal complexes by MALDI-TOF mass spectrometry. Clin. Microbiol. Infect. 2016, 22, e1-e161. [CrossRef] [PubMed]

18. Cox, C.R.; Jensen, K.R.; Saichek, N.R.; Voorhees, K.J. Strain-level bacterial identification by CeO2-catalyzed MALDI-TOF MS fatty acid analysis and comparison to commercial protein-based methods. Sci. Rep. 2015, 5, 1-13. [CrossRef]

19. Dubois, D.; Leyssene, D.; Chacornac, J.P.; Kostrzewa, M.; Schmit, P.O.; Talon, R.; Bonnet, R.; Delmas, J. Identification of a variety of Staphylococcus species by matrix-assisted laser desorption ionization-time of flight mass spectrometry. J. Clin. Microbiol. 2010, 48, 941-945. [CrossRef]

20. Dieckmann, R.; Helmuth, R.; Erhard, M.; Malorny, B. Rapid classification and identification of salmonellae at the species and subspecies levels by whole-cell matrix-assisted laser desorption ionization-time of flight mass spectrometry. Appl. Environ. Microbiol. 2008, 74, 7767-7778. [CrossRef]

21. Edwards-Jones, V.; Claydon, M.A.; Evason, D.J.; Walker, J.; Fox, A.J.; Gordon, D.B. Rapid discrimination between methicillin-sensitive and methicillin-resistant Staphylococcus aureus by intact cell mass spectrometry. J. Med. Microbiol. 2000, 49, 295-300. [CrossRef]

22. Wieme, A.D.; Spitaels, F.; Aerts, M.; De Bruyne, K.; Van Landschoot, A.; Vandamme, P. Effects of growth medium on matrix-assisted laser desorption-ionization time of flight mass spectra: A case study of acetic acid bacteria. Appl. Environ. Microbiol. 2014, 80, 1528-1538. [CrossRef]

23. Williamson, Y.M.; Moura, H.; Woolfitt, A.R.; Pirkle, J.L.; Barr, J.R.; Carvalho, M.D.G.; Ades, E.P.; Carlone, G.M.; Sampson, J.S. Differentiation of Streptococcus pneumoniae conjunctivitis outbreak isolates by matrix-assisted laser desorption ionization-time of flight mass spectrometry. Appl. Environ. Microbiol. 2008, 74, 5891-5897. [CrossRef] [PubMed]

24. Du, Z.; Yang, R.; Guo, Z.; Song, Y.; Wang, J. Identification of Staphylococcus aureus and determination of its methicillin resistance by matrix-assisted laser desorption/ionization time-of-flight mass spectrometry. Anal. Chem. 2002, 74, 5487-5491. [CrossRef] [PubMed]

25. Nix, I.D.; Idelevich, E.A.; Storck, L.M.; Sparbier, K.; Drews, O.; Kostrzewa, M.; Becker, K. Detection of Methicillin Resistance in Staphylococcus aureus From Agar Cultures and Directly From Positive Blood 
Cultures Using MALDI-TOF Mass Spectrometry-Based Direct-on-Target Microdroplet Growth Assay. Front. Microbiol. 2020, 11, 232. [CrossRef]

26. Wang, Y.R.; Chen, Q.; Cui, S.H.; Li, F.Q. Characterization of staphylococcus aureus isolated from clinical specimens by matrix assisted laser desorption/ionization time-of-flight mass spectrometry. Biomed. Environ. Sci. 2013, 26, 430-436. [PubMed]

27. Sung Kwon, S.; Kuk Hong, S.; Sook Kim, M.; Yong, D.; Lee, K. Performance of Matrix-Assisted Laser Desorption Ionization Time-of-Fight Mass Spectrometry for Rapid Discrimination of Methicillin-Resistant Staphylococcus aureus (MRSA): First Report of a Relation Between Protein Peaks and MRSA spa Type. Ann. Lab. Med. 2017, 37, 553-555. [CrossRef] [PubMed]

28. La Scola, B. Intact cell MALDI-TOF mass spectrometry-based approaches for the diagnosis of bloodstream infections. Expert Rev. Mol. Diagn. 2011, 11, 287-298. [CrossRef]

29. Goldstein, J.E.; Zhang, L.; Borror, C.M.; Rago, J.V.; Sandrin, T.R. Culture conditions and sample preparation methods affect spectrum quality and reproducibility during profiling of Staphylococcus aureus with matrix-assisted laser desorption/ionization time-of-flight mass spectrometry. Lett. Appl. Microbiol. 2013, 57, 144-150. [CrossRef]

30. Ferroni, A.; Suarez, S.; Beretti, J.L.; Dauphin, B.; Bille, E.; Meyer, J.; Bougnoux, M.E.; Alanio, A.; Berche, P.; Nassif, X. Real-time identification of bacteria and Candida species in positive blood culture broths by matrix-assisted laser desorption ionization-time of flight mass spectrometry. J. Clin. Microbiol. 2010, 48, 1542-1548. [CrossRef]

31. Giebel, R.A.; Fredenberg, W.; Sandrin, T.R. Characterization of environmental isolates of Enterococcus spp. by matrix-assisted laser desorption/ionization time-of-flight mass spectrometry. Water Res. 2008, 42, 931-940. [CrossRef] [PubMed]

32. Williams, T.L.; Andrzejewski, D.; Lay, J.O.; Musser, S.M. Experimental factors affecting the quality and reproducibility of MALDI TOF mass spectra obtained from whole bacteria cells. J. Am. Soc. Mass Spectrom. 2003, 14, 342-351. [CrossRef]

33. Vargha, M.; Takáts, Z.; Konopka, A.; Nakatsu, C.H. Optimization of MALDI-TOF MS for strain level differentiation of Arthrobacter isolates. J. Microbiol. Methods 2006, 66, 399-409. [CrossRef] [PubMed]

34. Shah, H.N.; Rajakaruna, L.; Ball, G.; Misra, R.; Al-Shahib, A.; Fang, M.; Gharbia, S.E. Tracing the transition of methicillin resistance in sub-populations of Staphylococcus aureus, using SELDI-TOF Mass Spectrometry and Artificial Neural Network Analysis. Syst. Appl. Microbiol. 2011, 34, 81-86. [CrossRef]

35. Walker, J.; Fox, A.J.; Edwards-Jones, V.; Gordon, D.B. Intact cell mass spectrometry (ICMS) used to type methicillin-resistant Staphylococcus aureus: Media effects and inter-laboratory reproducibility. J. Microbiol. Methods 2002, 48, 117-126. [CrossRef]

36. Mather, C.A.; Werth, B.J.; Sivagnanam, S.; Sengupta, D.J.; Butler-Wu, S.M. Rapid Detection of Vancomycin-Intermediate Staphylococcus aureus by Matrix-Assisted Laser Desorption Ionization-Time of Flight Mass Spectrometry. J. Clin. Microbiol. 2016, 54, 883-890. [CrossRef]

37. Quirino, A.; Pulcrano, G.; Rametti, L.; Puccio, R.; Marascio, N.; Catania, M.R.; Matera, G.; Liberto, M.C.; Focà, A. Typing of Ochrobactrum anthropi clinical isolates using automated repetitive extragenic palindromic-polymerase chain reaction DNA fingerprinting and matrix-assisted laser desorption/ionization-time-of-flight mass spectrometry. BMC Microbiol. 2014, 14, 74. [CrossRef]

38. Chung, C.-R.; Wang, H.-Y.; Lien, F.; Tseng, Y.-J.; Chen, C.-H.; Lee, T.-Y.; Liu, T.-P.; Horng, J.-T.; Lu, J.-J. Incorporating Statistical Test and Machine Intelligence into Strain Typing of Staphylococcus haemolyticus Based on Matrix-Assisted Laser Desorption Ionization-Time of Flight Mass Spectrometry. Front. Microbiol. 2019, 10, 2120. [CrossRef]

39. R Core Team. R: A Language and Environment for Statistical Computing; R Foundation for Statistical Computing: Vienna, Austria, 2017. 
40. Mellmann, A.; Cloud, J.; Maier, T.; Keckevoet, U.; Ramminger, I.; Iwen, P.; Dunn, J.; Hall, G.; Wilson, D.; LaSala, P.; et al. Evaluation of matrix-assisted laser desorption ionization-time-of-flight mass spectrometry in comparison to $16 \mathrm{~S}$ rRNA gene sequencing for species identification of nonfermenting bacteria. J. Clin. Microbiol. 2008, 46, 1946-1954. [CrossRef]

41. Valentine, N.; Wunschel, S.; Wunschel, D.; Petersen, C.; Wahl, K. Effect of culture conditions on microorganism identification by matrix-assisted laser desorption ionization mass spectrometry. Appl. Environ. Microbiol. 2005, 71, 58-64. [CrossRef]

42. Maier, T.; Klepel, S.; Renner, U.; Kostrzewa, M. Fast and reliable MALDI-TOF MS-based microorganism identification. Nat. Methods 2006, 3, i-ii. [CrossRef]

43. Alispahic, M.; Hummel, K.; Jandreski-Cvetkovic, D.; Nöbauer, K.; Razzazi-Fazeli, E.; Hess, M.; Hess, C. Species-specific identification and differentiation of Arcobacter, Helicobacter and Campylobacter by full-spectral matrix-associated laser desorption/ionization time of flight mass spectrometry analysis. J. Med. Microbiol. 2010, 59, 295-301. [CrossRef] [PubMed]

44. Gobom, J. Mass Spectrometry: MALDI. In Encyclopedic Reference of Genomics and Proteomics in Molecular Medicine; Springer: Berlin/Heidelberg, Germany, 2006; pp. 1023-1027.

45. Croxatto, A.; Prod'hom, G.; Greub, G. Applications of MALDI-TOF mass spectrometry in clinical diagnostic microbiology. FEMS Microbiol. Rev. 2012, 36, 380-407. [CrossRef] [PubMed]

46. Carbonnelle, E.; Beretti, J.L.; Cottyn, S.; Quesne, G.; Berche, P.; Nassif, X.; Ferroni, A. Rapid identification of staphylococci isolated in clinical microbiology laboratories by matrix-assisted laser desorption ionization-time of flight mass spectrometry. J. Clin. Microbiol. 2007, 45, 2156-2161. [CrossRef] [PubMed]

47. Bernardo, K.; Pakulat, N.; Macht, M.; Krut, O.; Seifert, H.; Fleer, S.; Hünger, F.; Krönke, M. Identification and discrimination of Staphylococcus aureus strains using matrix-assisted laser desorption/ionization-time of flight mass spectrometry. Pro. of the Prot. 2002, 2, 747-753.

48. Davis, J.A.; Farrah, S.R.; Wilkie, A.C. Selective growth of Staphylococcus aureus from flushed dairy manure wastewater using acriflavine-supplemented mannitol salt agar. Lett. Appl. Microbiol. 2006, 42, 606-611. [CrossRef]

49. Pomastowski, P.; Szultka-Młyńska, M.; Kupczyk, W.; Jackowski, M.; Buszewski, B. Evaluation of Intact Cell Matrix-Assisted Laser Desorption/Ionization Time of- Flight Mass Spectrometry for Capillary Electrophoresis Detection of Controlled Bacterial Clumping. J. Anal. Bioanal. Tech. 2015, S13, 008.

50. Hsieh, S.-Y.; Tseng, C.-L.; Lee, Y.-S.; Kuo, A.-J.; Sun, C.-F.; Lin, Y.-H.; Chen, J.-K. Highly Efficient Classification and Identification of Human Pathogenic Bacteria by MALDI-TOF MS. Mol. Cell. Proteom. 2008, 7, 448-456. [CrossRef]

51. Bright, J.J.; Claydon, M.A.; Soufian, M.; Gordon, D.B. Rapid typing of bacteria using matrix-assisted laser desorption ionisation time-of-flight mass spectrometry and pattern recognition software. J. Microbiol. Methods 2002, 48, 127-138. [CrossRef]

52. Šedo, O.; Sedláček, I.; Zdráhal, Z. Sample preparation methods for MALDI-MS profiling of bacteria. Mass Spectrom. Rev. 2011, 30, 417-434. [CrossRef]

53. Schober, P.; Schwarte, L.A. Correlation coefficients: Appropriate use and interpretation. Anesth. Analg. 2018, 126, 1763-1768. [CrossRef] [PubMed]

54. Gries, S.T. Quantitative Linguistics. In International Encyclopedia of the Social E Behavioral Sciences, 2nd ed.; Elsevier Inc., 2015; pp. 725-732. ISBN 9780080970875.

55. Jolliffe, I.T.; Cadima, J. Principal component analysis: A review and recent developments. Philos. Trans. R. Soc. A Math. Phys. Eng. Sci. 2016, 374, 20150202. [CrossRef] [PubMed]

56. Pomastowski, P.; Złoch, M.; Rodzik, A.; Ligor, M.; Kostrzewa, M.; Buszewski, B. Analysis of bacteria associated with honeys of different geographical and botanical origin using two different identification approaches: MALDI-TOF MS and 16S rDNA PCR technique. PLoS ONE 2019, 14. [CrossRef] [PubMed]

Sample Availability: Samples of the compounds obtained from the tested S. aureus strains are available from the authors.

Publisher's Note: MDPI stays neutral with regard to jurisdictional claims in published maps and institutional affiliations. 